A novel collaboration partner model based on the personal relationships of SNS, Yin, C., Yau, J. \& Tabata, Y. (2012), in Int. Conference on Active Media Technology, pp. 520-527.

\title{
A Novel Collaboration Partner Model Based on the Personal Relationships of SNS
}

\author{
Chengjiu Yin ${ }^{1}$, Jane Yin-Kim Yau ${ }^{2}$,Yoshiyuki Tabata ${ }^{1}$ \\ ${ }^{1}$ Research Institute for Information Technology, Kyushu University, \\ Hakozaki 6-10-1, Higashi-ku, Fukuoka, 812-8581 Japan. \\ \{yin, tabata\}@cc.kyushu-u.ac.jp \\ ${ }^{2}$ Dept of Computer Science, Malmö University, Sweden, \\ jane.yau@mah.se
}

\begin{abstract}
In this paper, we describe a novel model for locating appropriate 'helpers' for users based on the Chain of Friends $(\mathrm{CoF})$ personal relationship in a SNS system, in order to locate appropriate 'helpers' for different users. This model is called SESNMM (Search Engine for Social Networked Mobile Model) and allows individual users, located in remote locations, to participate in a collaborative online community, via our SESNMM-based system. Such typical helpers are willing to help other users solve their tasks/problems and it is intended that both the users and helpers gain knowledge from these interactive online sessions. We have applied this model for inviting PC members of an international conference - namely LTLE 2012. The results showed that our model is very effective for discovering collaboration partners, locating useful helpers, finding users with similar interests in order to create communities for providing future and longer-term helping and teaching exchange.
\end{abstract}

Keywords: Social Networking, Personal Relationship, Collaboration, Search Engine

\section{Introduction}

A social network has been defined by Boyd and Ellison (2008) as a web-based service which allows individuals to a) construct a profile within a bounded system, b) generate a list of other users with whom they share a connection, and c) view and connect to others via this list (e.g. a friend of a friend in Facebook). Different members within such a social networked site may have different mutual trust and closeness amongst one another. In particular, one of the fastest growing and most popular of social networking sites is the Social Networks Service (SNS). Such social interactions and access to social information resources on SNSs can be facilitated by mobile devices anytime and anywhere (Tan, Kinshuk, Jeng and Huang, 2010).

In this paper, we propose a novel model for locating appropriate 'helpers' for users based on the Chain of Friends (CoF) personal relationship in a SNS system, in order to locate appropriate 'helpers' for users. The model is called SESNMM (Search Engine for Social Networked Mobile Model). It builds on our previous work in foreign language learning exchange via SNS (Yin, Dong, Tabata and Ogata, 2012) 
where we developed a system for facilitating local Japanese students who wish to learn English, to find recommended native speakers across the globe that are willing to perform language exchange with them.

Every person/user in a social network has a personal relationship connection (e.g., "Friend", or "Friend of a Friend" in Facebook). In our SESNMM model, we depict these as shown in Figure 1 - direct Friend of User, or Acquaintances of A, Friend of B, or Acquaintances of C. These connections form the so-called "Chain of Friends" (CoF), from User to $D$.

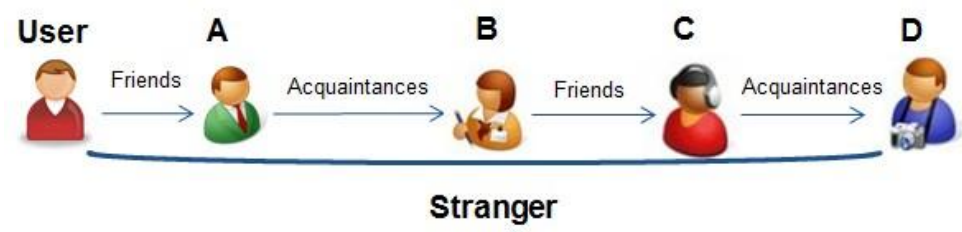

Figure 1. Chain of Friends.

Utilizing these personal relationships, our SESNMM model was designed to locate appropriate 'helpers' for individual users to help them with their tasks/ problems. For example, if the system can recommend a 'best' request $\mathrm{CoF}$ for users and locate a helper to the user, even if they are Strangers. This is a novel approach using personal relationships to get help from others even if they are Strangers.

This paper is organized as follows. In the next section, related work is presented. Then the recommendation algorithms we use in our system is described which is for finding appropriate 'helpers' and locating the 'best' request $\mathrm{CoF}$ to users. The process of the development of our SESNMM model is depicted. Finally, we present the conclusions and future work.

\section{Related Works}

An e-learning support system based on a location-based social network was developed by Kang and Choi (2011). These authors argued that the social interactions facilitated by such a system decrease the rate of drop-out in an e-learning course where there are normally minimal interactions between participants.

Relating to such systems is the so-called Help Network which was created before the born of the social networking technologies. A number of studies have demonstrated that one of the most effective channels for gathering information and expertise within an organization or institution is its informal network of collaborators, colleagues and friends (i.e. Help Network) (Eveland, Brown and Mattocks, 1994). Such a Help Network coupled with social networking technologies has huge potential in generating and sharing a rich wealth of information and learning resources as well as in providing a mechanism for instant real-time communication with others around the globe. Additionally, research suggests that social networks can potentially be useful for learners to solve problems because learners have access a) to many 'helpers' who can and may be willing to help them solve and complete the tasks, or b) lots of relevant and useful information/learning materials (El-Bishouty, Ogata, Rahman, and Yano, 2010). 
A novel collaboration partner model based on the personal relationships of SNS, Yin, C., Yau, J. \& Tabata, Y. (2012), in Int. Conference on Active Media Technology, pp. 520-527.

\section{Locating the Chain of Friends (CoF) in SESNMM model}

Our SESNMM model provides a connection between friends and acquaintances; this is also known as a Help Network system (Milgram, 1967). Our model can help users locate an appropriate collaborator/helper, who can in turn help them solve their tasks/problems, if any. If an appropriate helper is a Stranger without any connections, then locating a helper for this user may normally be a problem; this forms one of our research questions in this paper. In this section, we describe the algorithms we use in our model for locating appropriate 'helpers' and the 'best' request $\mathrm{CoF}$ to users.

\subsection{Algorithm for finding an appropriate collaborator/helper}

Our model uses the users' self-administered profiles (including personal information, study interests, schedules, and past actions) in order to locate an appropriate helper (who also has such a profile) who has the ability to solve the problem. Additionally, for each problem, the user enters some related keywords so that a match from the user and a helper can be found suited to the problem.

We designed a formula for calculating the appropriate degree. Consider that $n$ is the number of the keywords that the user inputs, and compare with the other person's profile, schedules, interests and actions, the number of the matched keywords is $n_{m}$. It is assumed that the Level of Matched Keywords (LMK) is calculated as follows:

$$
\left(L M K=\frac{n-n_{m}}{n}\right) \text {, where } \quad 0 \leq L M K \leq 1
$$

In case of LMK value is equal or close to zero, then the person will be recommended as an appropriate helper who is close to the user's request.

\subsection{Getting help from a Stranger}

We have previously carried out a survey that when we seek help/learning support from others and concluded that: 1) The more intimate the personal relationships are, the easier it is to get help/support; 2) The more simple things are, the easier it is to get help/support (Yin, Dong, Tabata and Ogata, 2012). Through this conclusion, we found a way for utilizing the $\mathrm{CoF}$ to get help from a stranger. Our model can recommend an appropriate request $\mathrm{CoF}$ to the user. In the case that there are many request $\mathrm{CoFs}$, the model recommends a 'best' request $\mathrm{CoF}$ according to the strength and personal relationship to the user.

For example, A is a Japanese person and studying Chinese. A wrote a blog in Chinese and wants someone to correct it for him, so he inputs the keyword "Language Learning, Chinese" and searches it on the system, and then the system becomes aware 
of the persons who have relations with the keyword and recommends an appropriate request $\mathrm{CoF}$ for $\mathrm{A}$.

As shown in the Figure 2, C and B can speak Chinese, $\mathrm{C}$ is a friend of $\mathrm{M}, \mathrm{B}$ is a internet friend of $\mathrm{M}$, and $\mathrm{M}$ is a friend of $\mathrm{A}$. There are 3 Request CoFs:

- For the case 1, A asks for the help to B or C directly. But as they are not friends or acquaintances, it is difficult that he would get help from $\mathrm{B}$ or $\mathrm{C}$.

- For the case 2, $\mathrm{C}$ is a friend of $\mathrm{M}$ and Chinese is her mother tongue.

- For the case 3, B is an internet friend of $\mathrm{M}$ and he is a Chinese learner.

Comparing the case 2 with the case 3 , the system recommends the case 3 for A. Then $\mathrm{A}$ asks $\mathrm{M}$ to introduce his friend $\mathrm{C}$ to him.

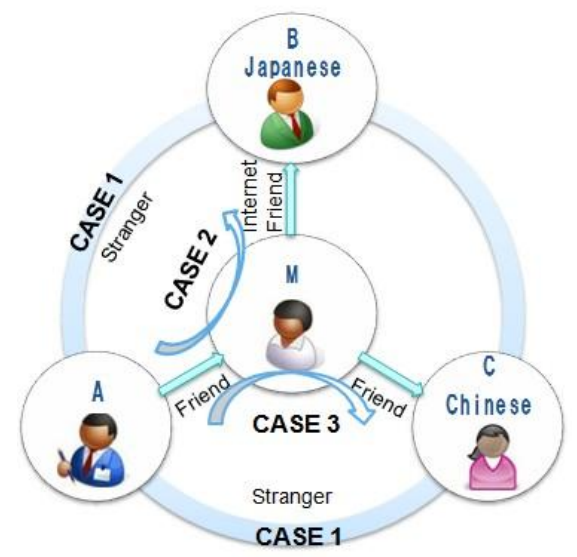

Figure 2. Request CoFs.

\subsection{Algorithm for recommending a 'best' request $\mathrm{CoF}$}

A 'best' CoF should not be only with a close personal relationship between these persons, but also the small number of the persons in the chain. It is the conditions to determine whether the $\mathrm{CoF}$ is appropriate or not.

The following table describes the categories of personal relationships, which we utilize in locating CoFs in our SESNMM mode. The table contains six categories of personal relationships, from an intimate relationship to an unfamiliar relationship, as shown in Table 1.

Table 1. Categories of Personal Relationships. 
A novel collaboration partner model based on the personal relationships of SNS, Yin, C., Yau, J. \& Tabata, Y. (2012), in Int. Conference on Active Media Technology, pp. 520-527.

\begin{tabular}{|l|c|l|}
\hline Relationship & Level & \multicolumn{1}{c|}{ Definition and Explanation } \\
\hline a) Family & 6 & They are family members such as father, mother or brother \\
\hline b) Relatives & 5 & They are very close to the leamer such as boy/girl friend, relatives or close friends like family. \\
\hline c) Friends & 4 & $\begin{array}{l}\text { They are persons whom the leaner has met and talked with him frequently such as friends, } \\
\text { classmate or teachers. }\end{array}$ \\
\hline d) Acquaintances & 3 & Acquaintances are persons whom the leaner has met and talked with him for a few times. \\
\hline e) Intemet friends & 2 & They are persons whom the leamer has never met before, but has talked for many times. \\
\hline f) Stranger & 1 & Strangers are persons whom the leamer has never met before. \\
\hline
\end{tabular}

According to the "six degrees of separation" theory (Milgram, 1967), we can know a social network typically comprises a person's set of direct and indirect personal relationships, and the length of the $\mathrm{CoF}$ is no more than six persons. We designed a formula for calculating $\mathrm{CoF}$ Adequacy (CoFA). Consider that $k$ is the number of the intermediaries in the $\mathrm{CoF}, m_{k}$ is level of the personal relationship which was selfadministered by the learner for the person $k$ in their profile, and we get a formula for calculating the $\mathrm{CoF}$ Adequacy (CoFA) in the following:

$$
\left(C o F A=\sum_{k=1}^{n}\left(\frac{k}{6} * \frac{6-m_{k}}{6}\right)\right), \text { where } 1 \leq m \leq 6, \quad n=\{1,2,3 \ldots\}, \text { and } \mathrm{k}=\{1,2,3 \ldots \mathrm{n}\}
$$

In case of CoFA value is more close to zero then the CoF is more appropriate, $\mathrm{n}$ is a natural number, and $\mathrm{k}$ is a natural number from 1 to $\mathrm{n}$.

\section{A Social Networked Collaboration Partner Model- SESNMM}

The main aim of our model is to facilitate collaboration and set up an online collaborative community amongst remote individual users via social networked mobile technologies.

Figure 3 shows our model depicting how individual users get help in an online community. 


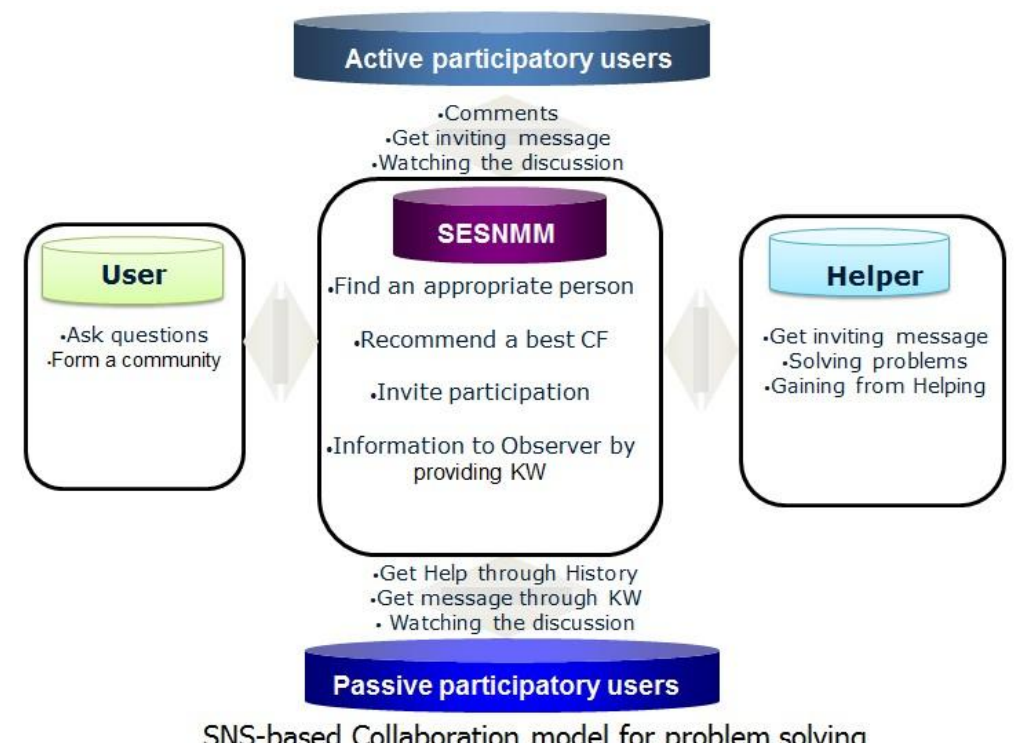

Figure 3: SESNMM- A Model for facilitating a collaborative online helping community

1. Overview: Figure 3 above illustrates that via the environment, how mutual help among users can be facilitated, and especially how users may find an appropriate partner(s) or 'helper(s)' in the online community for collaboration (or in order to solve some problem tasks collaboratively with others). An appropriate request $\mathrm{CoF}$ can be recommended upon their request by utilizing their personal relationships to determine which helpers can support the user to get help more easily. The system also sends messages to other persons, then a helping group is formed automatically to solve the problem. The methods of locating the CoF were explained above. Alternatively, the user can also create a community to discuss the problem. After the problem is solved, the helping group will be dissolved. The user will be alone again. The collaborative model is hence transformed back into the individual model.

On the left side of the diagram, one can see that in the original setting, a user is at home or in school individually. If he/she encounters a problem that he/she cannot resolve, then he/she can access a social networked environment to seek help from others online. The user's activity status is changed from individual to collaborative/group helping. Whereas users have the possibilities to gain help from peers in the offline setting, we argue that using such an online social networked model, users will have a larger and diverse set of 'peers/helpers' who may be willing to offer help, anytime, anywhere and on any topic.

2. Roles: There are four roles to the model - user, helper, participation, and observer. Each role has its own objectives which are fulfilled when the collaborative helping processes take place.

Users are these who have problems. Through the SESNMM based online system, they can solve the problems with others who may also correct their mistakes. They will acquire knowledge in the process. The users' mistakes/misconceptions can 
A novel collaboration partner model based on the personal relationships of SNS, Yin, C., Yau, J. \& Tabata, Y. (2012), in Int. Conference on Active Media Technology, pp. 520-527.

always be potentially corrected, which ensure that users acquire the correct knowledge.

Helpers are professors who are recommended by the system. Their roles are to help solve others' problems. This is gaining by helping which can enhance their understanding deeper by helping and teaching others.

Active participatory users are users who participate in the online community who want to enhance their understanding deeper by actively observing the discussion between the users and helpers. They can participate in the discussion if desired.

Passive participatory users are users who want to enhance their understanding deeper by actively observing the discussion between users and helpers. Users' mistakes can be regarded as good learning materials/lessons to learn from. These mistakes can help other users' in-depth understanding.

3. Problem-solving methods in SESNMM: There are two methods in which users can get help for solving their problems in our SESNMM model - one is via an indirect connection (i.e. it can be considered as a stranger) and the other one is via the online community.

1) The problem-solving method via an indirect connection works as follows. After a user has posted a problem in the SESNMM based system, the system automatically a) forms a learning group to solve this problem, b) extrapolates an appropriate helper who has the ability to solve the problem, and c) recommends a best Chain of Friends $(\mathrm{CoF})$ to help the user solve this problem. Additionally, the system invites some appropriate users to attend the group as participatory users, who also may have ability to solve the problem. These users may give comments relating to the problem from different points of views during the collaborative helping process. There may be others attending this helping session such as the Observers. Knowledge awareness functions are provided by the system so that users receive messages when there are helping sessions of topics related to their interests.

2) The problem-solving method via the online community works as follows. After a user has created his own helping community and posted his/her problem, the system automatically invites Participants as in the previous method. This community is not concerned with whether the problem can be solved completely but rather its focus is on facilitating users to actively participate, and gather more knowledge. Participants and Observers can give their opinions and comments according to their own experiences. All users can put forward their own point of views from different angles of the problem. Finally, the user collates these points of views to retrieve a solution to his problem.

\section{Conclusions and Future work}

We proposed a social networked collaboration model-SESNMM, which can facilitate collaboration and set up an online collaborative community amongst remote individual users via social networked mobile technologies. This model can locate appropriate 'helpers' for users based on the Chain of Friends (CoF) personal relationship in a SNS system, in order to locate appropriate 'helpers' for users. In this 
way, personal direct and indirect relationships can be utilized for pedagogical purposes, a network of friendships can be potentially enhanced, and knowledge sharing and creation can be supported and expanded.

Additionally, our model forms a SNS among users who have similar interests so that their own communities can be created for further and future learning and teaching exchange. We have applied this model for inviting PC members of the 2012 LTLE international conference. The results showed that our model is very effective for discovering collaboration partner.

\section{References}

Boyd, D. M. \& Ellison, N. B. (2008). Social network sites: Definition, history, and scholarship. Journal of Computer-Mediated Communication, vol. 13, no. 1, pp. 210-230.

El-Bishouty, M. M., Ogata, H., Rahman, S. \& Yano, Y. (2010) Social Knowledge Awareness Map for Computer Supported Ubiquitous Learning environment. Educational Technology \& Society, 13(4), pp. 2737.

Eveland, D. J., Brown, W. \& Mattocks, J. (1994) The Role of “Help Networks" in Facilitating Use of CSCW Tools. Proceedings of Computer Supported Cooperative Work, ACM Press, pp. 265-274.

Kang, J.-M. and Choi, S-Y. (2011) An e-learning support system based on location-based social network. International Journal of Advancements in Computing Technology, vol. 3, no. 7, pp. 55-63.

Milgram, S. (1967) The Small World Problem. Psychology Today, pp 60 - 67.

Tan, Q., Kinshuk, Jeng,Y-L \& Huang, Y.-M (2010) A collaborative mobile virtual system based on location based dynamic grouping. IEEE Int. Conference on advanced Learning Technologies, pp.16.18.

Yin, C., Dong, Y., Tabata, Y. \& Ogata, H. (2012) Recommendation of helpers based on personal connections in mobile learning. Int. Conference on Wireless, Mobile \& Ubiquitous Technologies in Education, pp.137-141. 